\title{
APPROXIMATION OF PERIODIC FUNCTIONS
} BY ZYGMUND MEANS IN ORLICZ SPACES

\author{
SADULLA Z. JAFAROV
}

Abstract. In the present work we investigate the approximation of the functions by Zygmund means in the Orlicz spaces $L_{M}(\mathbb{T})$ in the terms of the best approximation $E_{n}(f)_{M}$ and modulus of smoothness $\omega_{k}(\cdot, f)_{M}$.

Mathematics subject classification (2010): 41A10, 41A25, 42A10, 42B25, 46E30.

Keywords and phrases: reflexive Orlicz spaces; best approximation; trigonometric polynomials; $k-t h$ modulus of smoothness; Zygmund means of order $k$.

\section{REFERENCES}

[1] R. Akgun, D.M. IsRafiLov, Approximation by interpolating polynomials in Smirnov-Orlicz class, J. Korean Math. Soc. 43, 2 (2006), 413-424.

[2] R. Akgun, D. M. IsRafilov, Approximation and moduli of fractional orders in Smirnov-Orlicz classes, Glas. Mat. Ser. III 43 (63), 1 (2008), 121-136.

[3] R. Akgun, D. M. IsRafilov, Simultaneous and converse approximation theorems in weighted Orlicz spaces, Bull. Belg. Math. Soc. Simon Stevin 17 (2010), 13-28.

[4] R. AKGUN, Improved converse theorems and fractional moduli of smoothness in Orlicz spaces, Bull. Malays. Math. Sci. Soc, (2) 36 (2013), 49-62.

[5] S. P. Baiborodov, Approximation of functions by Vallée-Poussin sums, Mat. Zametki, 27, 1 (1980), 33-48 (in Russian).

[6] A. Bottcher And Yu. I. Karlovich, Carleson curves, Muckenhoupt weights and Teoplitz operators, Birkhauser - Verlag, (1997).

[7] D. W. Boyds, Indices for the Orlicz spaces, Pacific J. Math. 38, 2 (1971), 315-323.

[8] V. G. GAVRILYUK, Linear methods of summing Fourier series and best approximation, Ukr. Math. J. 15, 4 (1963), 412-417.

[9] A. Guven, D. M. IsRafilov, Polynomial approximation in Smirnov-Orlicz classes, Comput. Meth. Funct. Th. 2, 2 (2002), 509-517.

[10] A. Guven, D. M. IsRafilov, Approximation by means of Fourier trigonometric series in weighted Orlicz spaces, Adv. Stud. Contemp. Math. (Kyundshang), 19, 2 (2009), 283-295.

[11] N. A. IL'YASOV, Approximation of periodic functions by Zygmund means, Mat. Zametki, 39, 3 (1986), 367-382 (in Russian).

[12] N. A. IL'YASOV, On the order of approximation in the uniform metric by Fejerr-Zygmund means in the classes $E_{p}[\varepsilon]$, Mat. Zametki, 69, 5 (2001), 679-687 (in Russian).

[13] D. M. IsrafiLov, R. Akgun, Approximation in weighted Smirnov-Orlicz classes, J. Math. Kyoto Univ. (JMKYAZ) 46, 4 (2006), 755-770.

[14] D. M. Israfilov, A. Guven, Approximation by trigonometric polynomials in weighted Orlicz spaces, Studia Math. 174, 2 (2006), 147-168.

[15] S. Z. Jafarov, Approximation by rational functions in Smirnov-Orlicz classes, J. Math. Anal. Appl. 379 (2011), 870-877.

[16] S. Z. Jafarov, The inverse theorem of approximation of the function in Smirnov-Orlicz classes, Math. Inequal. Appl. 12, 4 (2012), 835-844.

[17] S. Z. Jafarov, J. I. MamedKhanov, On approximation by trigonometric polynomials in Orlicz. spaces, Georgian Math. J. 19, 4 (2012), 687-695. 
[18] S. Z. JAfArov, Approximation by Fejér sums of Fourier trigonometric series in weighted Orlicz spaces, Hacet. J. Math. Stat. 42, 3 (2013), 259-268.

[19] S. Z. JAFAROV, Linear methods for summing Fourier series and approximation in weighted Lebesgue with variable exponents, Ukrainian Math. J. 66, 10 (2015) 1509-1518.

[20] S. M. NiKOLS KI, On linear methods of summation of Fourier series, Akad. Nauk SSSR ser. Math. 12 (1948), 259-278 (in Russian).

[21] M. A. Krasnoselski, YA. B. Rutickit, Convex Functions and Orlicz Spaces, P. Norrdhoff Ltd., Groningen, 1961.

[22] V. M. Kokilashvili, On analytic functions of Smirnov-Orlicz classes, Studia Math. 31 (1968), 4359.

[23] V. M. KoKilashVili, On approximation of periodic functions, Trudy Tbiliss. Mat. Inst. im. Razmadze Akad. Nauk Gruzin. SSR 34 (1968), 51-81 (in Russian).

[24] V. M. Kokilashvili, S. G. Samko, Operators of harmonic analysis in weighted spaces with nonstandard growth, J. Math. Anal. Appl. 352 (2009), 15-34.

[25] V. M. KoKILASHVILI, Ts. Ts ANAVA, On the norm estimate of deviation by linear summability means and an extension of the Bernstein inequality, Proc. A. Razmadze Math Inst. 154 (2010), 144-146.

[26] V. M. Kokilas vili, Ts. Ts anava, Approximation by linear summability means in weighted variable exponent Lebesgue spaces, Proc. A. Razmadze Math. Inst. 154 (2010), 147-150.

[27] A. YU. KARLOVICH, Algebras of Singular integral operators with piecewise continuous coefficients on reflexive Orlicz spaces, Math. Nachr. 179 (1996), 187-222.

[28] W. Matuszewska, W. Orlicz, On certain properties of $\phi$-functions, Bull. Acad. Polon. Sci., Ser. Math. Aster. et Phys. 8 (1960), 439-443.

[29] L. Maligranda, Indices and interpolation, Dissertation Math. 234 (1985).

[30] Le.Yu. Ovsi, A. S. SERdyuk, Approximation of continuous periodic functions by de la ValléePoussin sums, Zb. Pr. Inst. Mat. NAN Ukr. 8, 1 (2011), 151-161 (in Russian).

[31] V. G. Ponomarenko, Approximation of periodic functions in an Orlicz space, Sibirsk. Mat. J. 6 (1966), 1338-1346 (in Russian).

[32] A.-R. K. Ramazanov, On approximation by polynomials and rational functions in Orlicz spaces, Anal. Math. 10 (1984), 117-132.

[33] R. RYAn, Conjugate functions in Orlicz spaces, Pacific J. Math, 13 (1963), 1371-1377.

[34] M. M. RaO, Z. D. REN, Theory of Orlicz spaces, Marcel Dekker, New York, 1991.

[35] K. Runovs Ki, On Jackson type inequality in Orlicz classes, Rev. Mat. Complut. 14 (2001), $395-404$.

[36] S. B. Stechkin, The approximation of periodic functions by Fejér sums, Trudy Math. Inst. Steklov, G2 (1961), 522-523 (in Russian).

[37] S. B. Stechkin, Approximation of functions by Vallée- Poussin sums, Mat. Zametki 27, 1 (1980), 33-48 (in Russian).

[38] A.S. Serdyuk, Le. Yu. Ovsi, A. P. Musienko, Approximation of classes of analytic functions by de la Vallée-Poussin sums in uniform metric, Rend. Mat. Appl., Ser. VII 32 (2012), 1-15.

[39] M. F. Timan, Some linear summation processes for Fourier series and best apptroximation, Dokl. Akad. Nauk SSSR 145 (1962), 741-743.

[40] M. F. Timan, Best approximation of a function and linear methods of summing Fourier series, Izv. Akad. Nauk SSSR Ser: Math. 29 (1965), 587-604 ( in Russian).

[41] M. F. TIMAN, The approximation of continuous periodic functions by linear operators which are constructed on the basis of their Fourier series, Dokl. Akad. Nauk SSSR 181 (1968), 1339-1342 (in Russian).

[42] G. WU, On approximation by polynomials in Orlicz spaces, Approx. Theory Appl. 7, 3 (1991), $97-$ 110.

[43] A. A. ZaKhaRov, Bound on deviations of continuous periodic functions from their de la ValléePoussin sums, Mat. Zametki, 3, 1 (1968), 77-84 (in Russian). 